\title{
Dynamic measuring methods: a review
}

\author{
Aleksandr L. Shestakov ${ }^{1}$ \\ ${ }^{1}$ South Ural State University, Lenina, 76, Chelyabinsk, Russia
}

\section{ABSTRACT}

The paper presents an overview of the directions in the development of dynamic measurement methods. First, the concept of dynamic measurements and a short historical background are outlined. The author describes several types of dynamic characteristics of measuring equipment, which fully characterise their dynamic properties. The next part is devoted to an analysis of the types of dynamic errors and their corrections. In conclusion, an overview of the identification of dynamic characteristics is given.

\section{Section: RESEARCH PAPER}

Keywords: Dynamic measurements; dynamic error analysis; restoration of a dynamically distorted signal; regularisation; evaluation of the dynamic characteristics of measuring equipment

Citation: Aleksandr L. Shestakov, Dynamic measuring methods: a review, Acta IMEKO, vol. 8, no. 1, article 10, March 2019, identifier: IMEKO-ACTA-08 (2019)-01-10

Section Editor: Franco Pavese, Italy

Received March 23, 2018; In final form May 31, 2018; Published March 2019

Copyright: (C) 2019 IMEKO. This is an open-access article distributed under the terms of the Creative Commons Attribution 3.0 License, which permits unrestricted use, distribution, and reproduction in any medium, provided the original author and source are credited.

Funding: The work was supported by Act 211, Government of the Russian Federation, contract № 02.A03.21.0011.

Corresponding author: Aleksandr Shestakov, e-mail: a.l.shestakov@susu.ru

\section{THE CONCEPT OF DYNAMIC MEASUREMENTS}

The term 'dynamic measurements' refers to measurements whereby the output signal is significantly different to the measuring input signal due to the inertia lag of the measuring equipment or measuring system. Such situations occur when impulse or other quick-changing actions are measured. A sensor, or the whole test facility, can act as measuring equipment. A common example of such measurements is an impulse pressure measurement $P(t)$ (Figure 1).

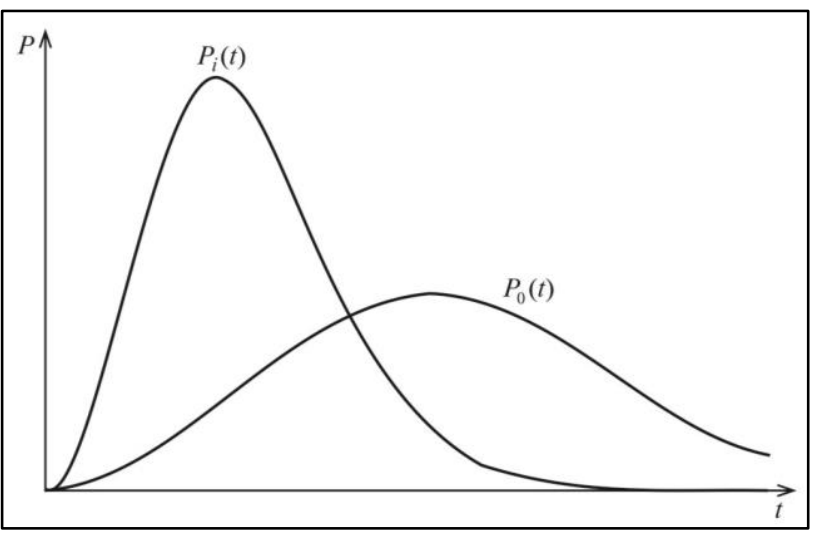

Figure 1. Dynamic measuring of impulse pressure: $P_{i}=$ measuring input signal, $P_{0}=$ observed output signal
As a rule, the value of the dynamic error is a time function. It is significantly different to the measurement uncertainty in static measurements. In dynamic measurements, errors of tens of per cents are common.

\section{SHORT HISTORICAL BACKGROUND}

The pioneering work on dynamic measurement is the study of the French physicist Cornu [1], published in 1887. In this work, he studied the motion of a galvanometer and associated the value of the damping factor with the physical parameters of the device.

Later on, the inventor of an oscilloscope, French engineer Blondel [2], considered dynamic models of some of its modules (1893). Also, the work of D. I. Mendeleev [3], published in 1897 and focused on accurate weighing on the unsteady laboratory balance should be referred to the first academic papers. In this work the great Russian chemist without using differential equations found formulas for accurate weighing in the context of the swing of laboratory balance.

The work of A. N. Krylov [4], wherein a device for measuring mine explosion pressure was examined, can be considered as a starting point in the development of the theory of dynamic measurement. Measuring error was studied as a time function for the first time. V. A. Granovskii had a key role in the development of the theory of dynamic measurement, and 


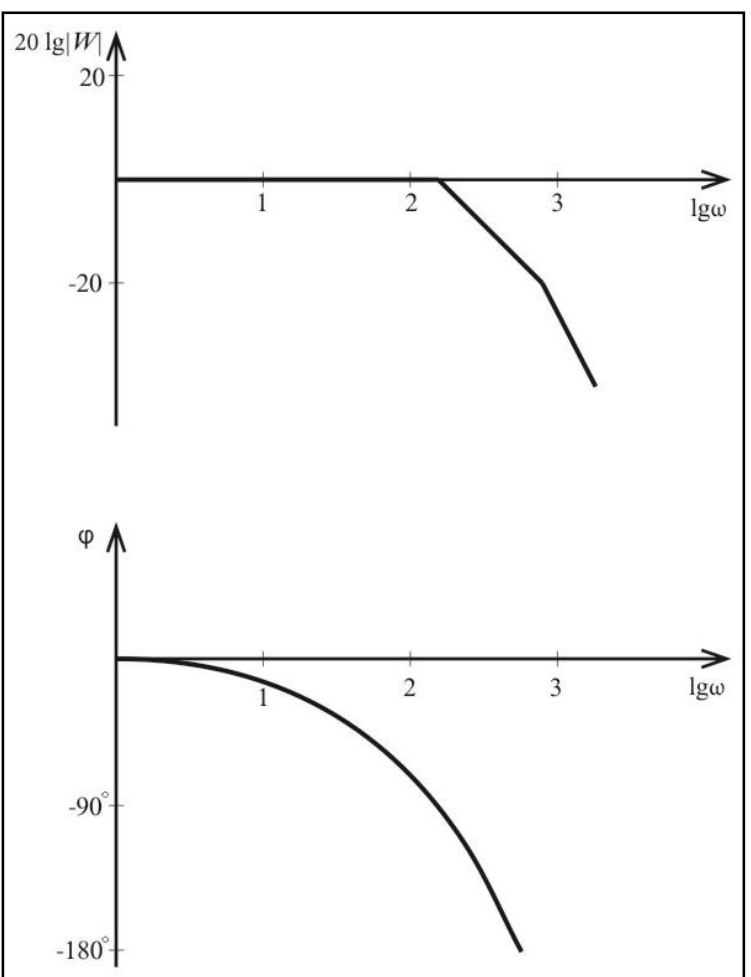

Figure 2. Amplitude- and phase-frequency characteristics of measuring equipment

in his book [5], he presented all the main parts of this theory: the determination of the dynamic characteristics of measuring equipment, analysis of dynamic errors, dynamic error correction, and issues of dynamic measurement uniformity.

\section{DYNAMIC CHARACTERISTICS OF MEASURING EQUIPMENT}

There are several types of the dynamic characteristics of measuring equipment, which fully characterise its dynamic properties. The transfer function of the measuring equipment is

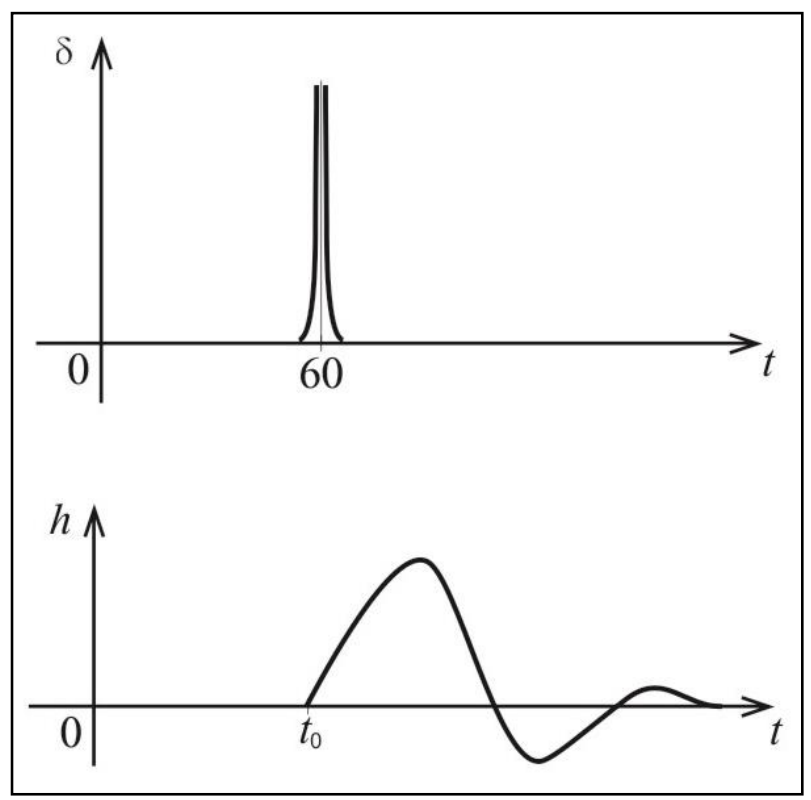

Figure 3. Impulse transient function of the measuring equipment.

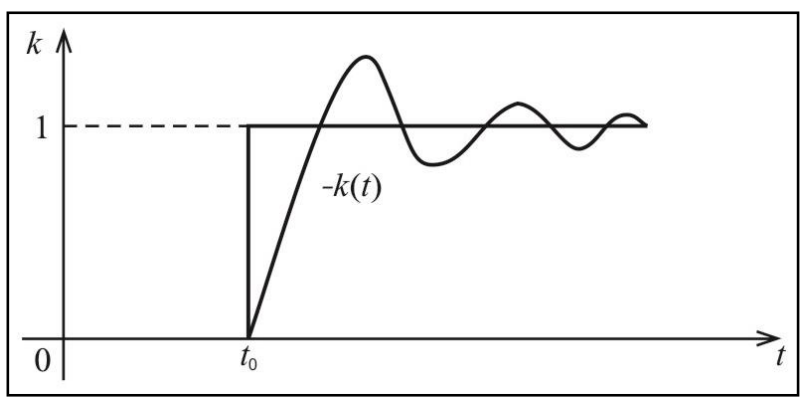

Figure 4. Transient function of the measuring equipment.

$W(p)=\frac{y(p)}{u(p)}=\frac{p^{m}+b_{m-1} p^{m-1}+\cdots+b_{1} p+b_{0}}{p^{n}+a_{n-1} p^{n-1}+\cdots+a_{1} p+a_{0}}$,

where $u(p)$ and $y(p)$ are Laplace transforms of input and output signals $m<n$, and its discrete analogue is:

$W(z)=\frac{y(z)}{u(z)}=\frac{z^{l}+r_{l-1} z^{l-1}+\cdots+r_{1} z+r_{0}}{z^{k}+q_{k-1} z^{k-1}+\cdots+q_{1} z+q_{0}}$

$Z$ is a delay operator. The frequency characteristics of the measuring equipment, amplitude-frequency and phasefrequency characteristics are illustrated in Figure 2.

The impulse transient function $h(t)$ (Figure 3 ) is a response of the measuring equipment to the $\delta$-impulse of the infinitely small duration, in terms of time and an infinitely large amplitude

$\int_{0}^{\infty} \delta(t) d t=1$

The transient function $k(t)$ (Figure 4) is a response of the measuring equipment to the unit step input.

All these characteristics fully characterise the dynamic properties of the measuring equipment, and from this perspective, they are equivalent. Furthermore, there are methods for converting one characteristic into another. Theoretical methods for defining dynamic characteristics have been quite fully developed in terms of identifying automatic control systems. Moreover, there is no differential difference between the automatic control system and the dynamic measuring system. However, the dynamic characterisation of the measuring equipment is a complex problem. In the passport data provided by the sensor manufacturers, the presence of dynamic characteristics is still the exception rather than the norm. For complex dynamic measuring facilities, this process requires special experiments.

\section{ANALYSIS OF DYNAMIC MEASURING ERRORS}

After the information on dynamic characteristics of measuring equipment is obtained, the next question is: What error occurs when taking measurements in dynamic mode? We can single out several approaches to the analysis of dynamic measuring errors. For the first, the deterministic approach, a structural diagram of the dynamic error analysis can be represented in the following way (Figure 5):

The Laplace transforms of dynamic errors take the form

$\varepsilon(p)=u(p)[W(p)-1]$

In fact, the dynamic error through the inverse Laplace transform is measured as follows 


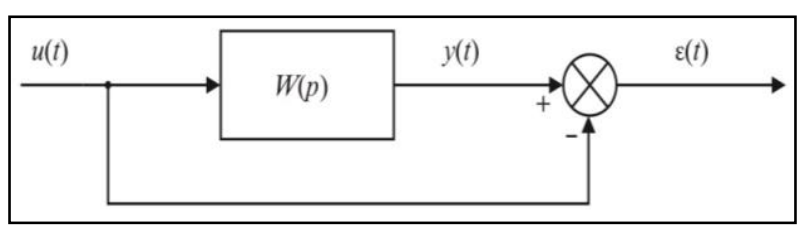

Figure 5. Structural diagram of forming dynamic errors in the deterministic case.

$\varepsilon(t)=L^{-1}\{u(p)[W(p)-1]\}$,

and through the solution of an integral equation

$\varepsilon(t)=\int_{0}^{\infty} h(t-\tau) u(\tau) d t-u(t)$,

where $W(p)$ and $h(t-\tau)$ are the transfer function and the impulse transient function of measuring equipment respectively.

Equation (4) and equation (5) show that the dynamic error of measurements is the time function and depends both on the dynamic properties of the measuring equipment and on the measuring signal.

The analytical expression for the dynamic errors of the measuring step signal for a measuring transducer of arbitrary order was outlined by V. A. Granovskii [5]. In a general case in which the input of the measuring system is affected by the input signal of the Laplace transform, which can be represented as $u(p)=u_{1}(p) / u_{2}(p)$. The measuring system has the transfer function $W(p)=M(p) / D(p)$, where $M(p)$ and $D(p)$ are polynomials of $m$ and $n$ order, respectively, and $K(0)=$ $M(0) / D(0)$. The dynamic error, in steady-state conditions, takes the form

$\varepsilon(t)=\sum_{k=1}^{v} \frac{u_{1}\left(\rho_{k}\right)}{u_{2}^{\prime}\left(\rho_{k}\right)}\left[\frac{\prod_{i=1}^{m}\left(1-\frac{\rho_{k}}{\gamma_{i}}\right)}{\prod_{i=1}^{n}\left(1-\frac{\rho_{k}}{\lambda_{i}}\right)}-1\right] e^{\rho_{k}(t)}$

where $\lambda_{1}, \ldots \lambda_{n}$ and $\gamma_{1}, \ldots \gamma_{m}$ are transfer function poles and zeros. $\rho_{k}$ is a pole of the Laplace transform of the input signal $u$ [6]. In the same work, dependencies for determining the poles and zeros of the measuring system, providing the required maximum allowable errors to measure linear, parabolic, and exponential signals, are obtained.

More complex signals can be approximated by a combination of simpler signals and taking into account nonzero initial conditions when they are joined. However, analytical dependencies are lengthy. The mathematical simulation method is more preferable for analysing dynamic errors in such a case. This approach is useful for the analysis of dynamic errors when signals of the known form are measured.

[7] presents the expressions of the dynamic errors of the measuring system used when periodic square impulse and trapezoidal wave signals are measured. The transition function $h(t)$ is used here as a characteristic of the dynamic properties of the measuring system.

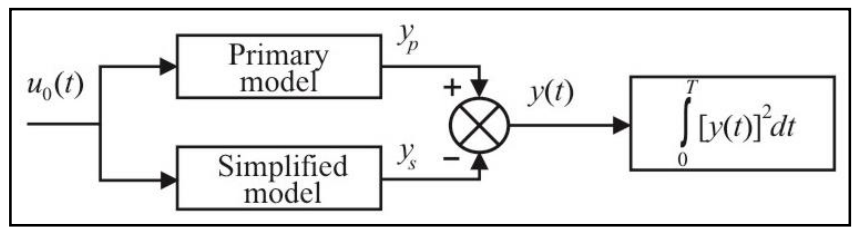

Figure 6. Block diagram for determining the integral square error.

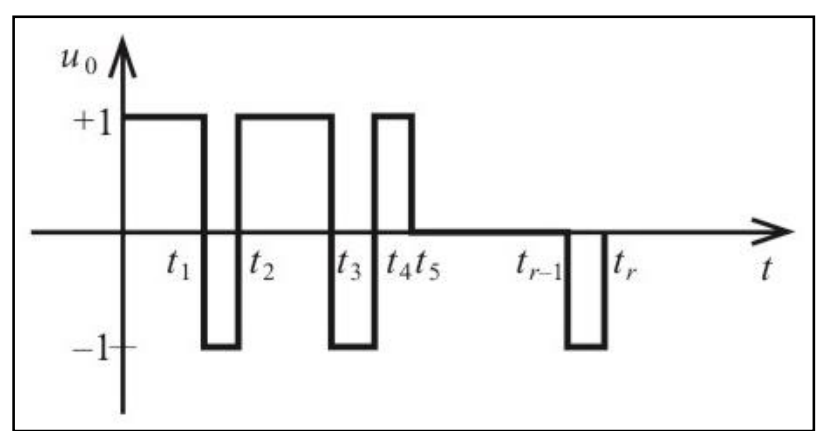

Figure 7. Input signal maximising the integral square error.

There is also an approach whereby a dynamic error is determined as an integral in some period of observing an integral square error. Thus, [8] analyses the method for decreasing an order of the dynamic system based on the minimisation of a quadratic error criterion for a simplified system with regards to a high-order system.

The error formation scheme is shown in Figure 6.

The method for determining a signal $u_{0}(t)$ switching from +1 to -1 at the moments $t_{1}, t_{2}, \ldots t_{r}$ (Figure 7), maximising the square error on the interval $[0, T]$, was suggested.

The results of this work can be considered as a method for finding a signal that generates the maximum dynamic error of the measuring system.

[9] considers a method for obtaining a switching input signal, limited in the value $|u(t)| \leq a$, whose speed is also limited. $|\dot{u}(t)| \leq \theta, t \in[0, T]$ maximises the integral square error of the difference between the signals of the measuring system and its simplified dynamic model. The method requires the solution of a set of complicated integral-convolution equations. As the author notes, applying this method, it is difficult to receive a signal with more than 25 switching operations.

[10] suggests a method for generating a switching signal on the basis of wavelet transform. The measured input signal has limitations in amplitude and the input change rate (Figure 8).

The method generates a switching signal that maximises the integral square error of the dynamic measuring system. The switching points are specified by the genetic algorithm.

Based on the approach by which the measured input signal (which provides the maximum integral square error of the dynamic measuring system) is generated, an attempt to create a hierarchy of dynamic measuring systems according to their dynamic accuracy was made in [11]. The diagram for determining dynamic accuracy is suggested in Figure 9.

Here, $K_{x}(p)$ and $K_{s}(p)$ are the transfer functions of the

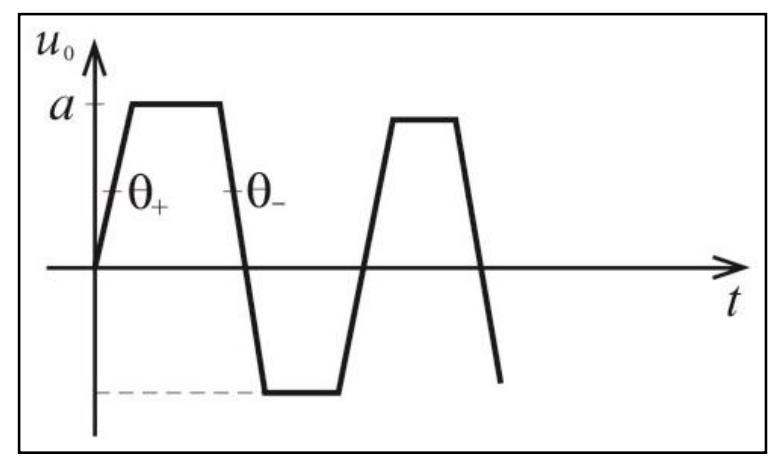

Figure 8. Input signal maximising the integral square error 


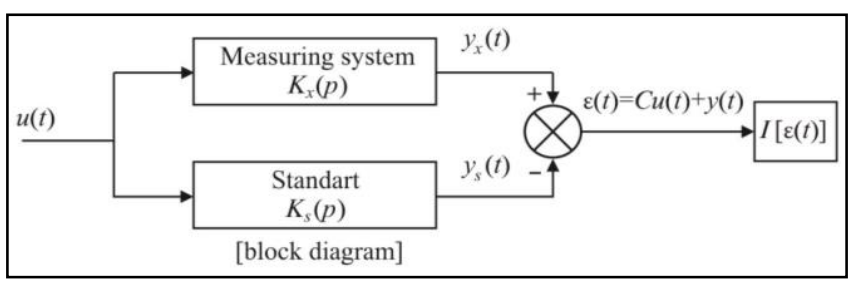

Figure 9. Block diagram for determining dynamic errors of the system

measuring system and a standard system with the desired dynamic characteristics. The system error has two components.

The coefficient

$c=\lim _{p \rightarrow \infty}\left[K_{x}(p)-K_{s}(p)\right]$

specifies a steady-state component of the error $c u(t)$, and

$y(t)=\int_{0}^{t} k(t-\tau) u(\tau) d t$

where $k(t-\tau)$ is an impulse transient function of the difference between the systems $K_{x}(p)-K_{s}(p)$.

A criterion for the dynamic accuracy of the measuring system is proposed on the basis of the quadratic criterion

$I=\int_{0}^{T}[c u(t)+y(t)]^{2} d t$

to use its maximum value

$\left.\max I[\varepsilon(t)]=\max _{t \in[0, T] u \in[U]} \max _{u} I \varepsilon(t)[u(t), c, k(t), T]\right\}$

The attempt to classify (create a hierarchy) dynamic measuring systems by dynamic errors is very useful. However, it has not been as successful as the establishment of measuring equipment in the case of static measurements by measurement uncertainty and accuracy classes. It is not clear what is considered as a standard measuring system. The situation is logical. As shown in [9], the measured signal $u(t)$, maximising the integral square error, is disruptive. Furthermore, finding it is highly complicated, especially for high-order systems. The interval of integration should also be compared with the dynamic properties of the measuring system. Nevertheless, the problem statement is promising.

The third approach to the analysis of dynamic errors is based on consideration of the measured signal as random with the known spectral density. Then, the structural diagram for estimating the measurement errors can be presented in the following form (Figure 10).

In Figure 10, $W(j \omega)$ is a frequency characteristic of the dynamic measuring system. $S_{u}(\omega), S_{n}(\omega), S_{w}(\omega), S_{y}(\omega)$, $S_{\varepsilon}(\omega)$ are spectral densities of the input signal $u(t)$; input and output noise $n(t)$ and $w(t)$; the output signal $y(t)$, and the error $\varepsilon(t)$. The spectral densities, even the function in the form

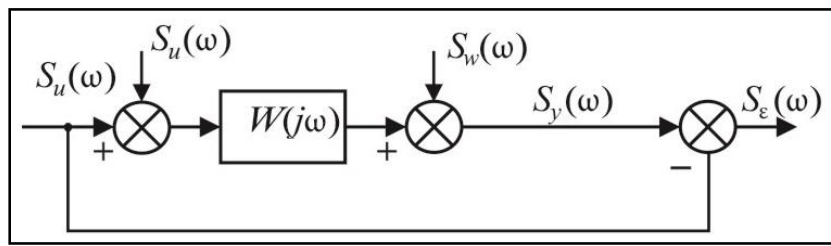

Figure 10. Structural diagram of the formation of dynamic errors in the case of a random signal.

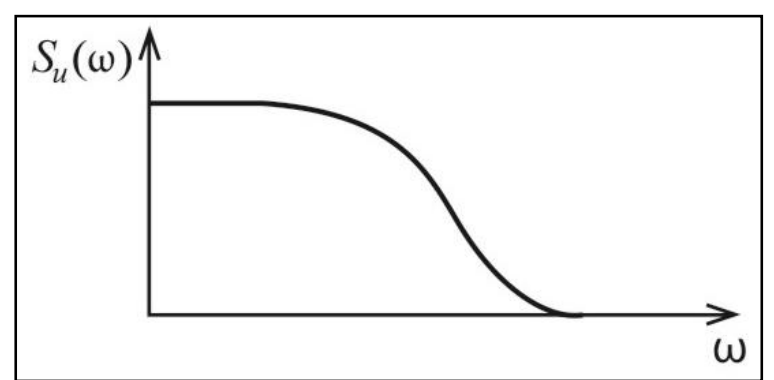

Figure 11. Typical characteristic of the spectral density of the measured signal.

of fractional rational functions, can be represented as [12], [13]

$S_{u}(\omega)=\frac{b_{0}+b_{1} \omega^{2}+\cdots+b_{m} \omega^{2 m}}{a_{0}+a_{1} \omega^{2}+\cdots+a_{n} \omega^{2 m}}$.

The typical characteristic of the spectral density of the measured input signal indicates that this signal always has a restricted frequency spectrum (Figure 11). Taking into account the mutual uncorrelatedness of signals $u(t), n(t)$, and $w(t)$, the spectral density of the error takes the form

$S_{\varepsilon}(\omega)=|1-W(j \omega)|^{2} S_{u}(\omega)+|W(j \omega)|^{2} S_{u}(\omega)+S_{w}(j \omega)$

The average value of the squared error is calculated by the spectral density

$\bar{\varepsilon}^{2}=\frac{1}{2 \pi} \int_{-\infty}^{\infty} S_{\varepsilon}(\omega) d \omega$.

\section{DYNAMIC ERROR CORRECTION}

By this term, we refer to the decrease in dynamic measuring errors by mathematical processing of the measurement results using the knowledge of dynamic characteristics of the measuring system. There is also another name for this process: the restoration of a dynamically distorted signal.

The first approach, which results in a number of methods for solving this problem, is based on the description of the process of dynamic measurements by the Volterra equation of the first kind. The impulse transient function $h(t-\tau)$ is used as a dynamic characteristic of the measuring equipment (Figure 12).

The measured input signal $u(t)$ is connected with the observed output signal $y(t)$ by the integral equation

$y(t)=\int_{T_{1}}^{t} h(t-\tau) u(\tau) d \tau$,

where $T_{1} \leq t \leq T_{2}$. In this case, $T_{1}$ can be equal to 0 or $-\infty$, and $T_{2}=\infty . h(t-\tau)$ is an impulse transient function of the measuring equipment. As the variable $t$ is time, the output signal cannot appear before the input one. That is why a requirement of physical realisability should be met:

$$
h(t-\tau)=0 \text { in case of } t<\tau .
$$

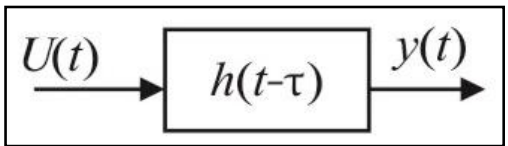

Figure 12. Block diagram of the measuring equipment represented by the impulse transient function. 
To restore the measured signal $u(t)$, it is necessary to solve equation (12). The direct method for solving equation (12) is based on replacement of the integral by the sum [14]:

$\sum_{j=1}^{i} A_{j} h_{i j} u_{j}=y_{i}$

are coefficients of the quadrature formula. There are many such formulas [15], [16]. However, a general feature of this approach is the impossibility of the direct determination of $u_{1}$ for further development of a recurrent algorithm. To find it, it is necessary to differentiate $y(t)$ at the starting point

$u_{1}=\frac{y^{\prime}\left(T_{1}\right)}{h_{11}}$.

In the presence of noise in the output signal, this leads to a significant error.

There are many possible methods of solving equation (12). In [17], it is suggested to approximate the output signal $y(t)$ and the impulse transient function $h(t-\tau)$ by a certain type of function. Taking into account a damped oscillating nature of the studied processes, we can approximate

$y(t)=e^{-\beta|t|} \cdot \cos \omega t$

$h(t-\tau)=e^{-\alpha|t-\tau|} \cdot \cos (v(t-\tau))$,

where $\beta, \alpha, \omega, v$ are approximation parameters. Then, equation (12) can be solved by simple numerical or even analytical methods.

One method for solving equation (12) is based on the series expansion of the desired signal $u(t)$ by eigenfunctions $\varphi_{i}(t)$ of the impulse transient function $h(t-\tau)$ [18], which satisfies the equation

$\int_{-a}^{a} h(t-\tau) \varphi_{i}(\tau) d \tau=\lambda_{i} \varphi_{i}(t)$

where $\lambda_{i}$ is an eigenvalue of the function $h(t-\tau)$. Then, $u(t)$ is expressed in terms of the series sum, as follows:

$u(t)=\lim _{N \rightarrow \infty} \sum_{i=0}^{N} S_{i} \varphi_{i}(t)$.

The recurrent algorithm for calculating the measured signal is suggested in [19]:

$u_{n+1}(t)=y(t)+u_{n}(t)-\int_{T_{1}}^{t} h(t-\tau) u_{n}(\tau) d \tau$,

where $u_{0}(t)=0 ; u_{1}(t)=y(t)$.

The common feature of the abovementioned methods is the high sensitivity of solving the Volterra integral equation to noise, which is always present in the output signal of the measuring equipment. This is due to the incorrectness of the task in restoring the dynamically distorted signal. One of the first works, which has been devoted to the peculiar features of dynamic measurement, is the work of G. N. Solopchenko [20].

In [21], the integral equation is solved taking into account the output noise of the measuring equipment. The integral equation (12) is approximated by a discrete equation, which in matrix form, is written as $\bar{y}=H \bar{u}+\bar{z}$, where

$\bar{y}=[y(0), y(1), \ldots, y(M-1)]^{T}$

$$
\begin{aligned}
& \bar{u}=[u(0), u(1), \ldots, u(N-1)]^{T} \\
& \bar{z}=[z(0), z(1), \ldots, z(M-1)]^{T} .
\end{aligned}
$$

It is assumed that the output noise $z$ of the measuring equipment is a Gaussian probability distribution with the average $E\{\bar{z}\}=0$ and the known covariance matrix $R_{z}=$ $E\left\{\bar{z} \bar{Z}^{T}\right\}$. Minimising the mean-square error by the least squares method, the values of the measured signal takes the form

$\bar{u}=\left(H^{T} R_{z}^{-1} H\right)^{-1} H^{T} R_{z}^{-1} \bar{y}$.

The method is quite simple and takes into account the noise assuming it is Gaussian. However, we note that the measurement error increases with time, and it does not quite solve the problem of noise control.

When the dynamically distorted signal is restored, the noise present in the output signal is amplified. The desire to weaken the influence of this phenomenon has led to the development of methods for regularising the solution to equation (12). The work of A. N. Tikhonov [22] has been highly valuable in the development of these methods. The principle of the method for producing regularised solutions of equation (12) is as follows. Let the output signal of the measuring equipment be represented in the following form:

$y(t)=y_{T}(t)+v(t)$,

where $v(t)$ is measurement noise; $y_{T}(t)$ is an output signal of the measuring equipment without noise at its output. Applying the Fourier transform to equation (12), reduced to the infinite limits of integration, we can write

$u(\omega) \cdot H(\omega)=y_{T}(\omega)+v(\omega)$,

where $u(\omega), y_{T}(\omega), v(\omega)$ is the Fourier transform of the corresponding signals, and $H(\omega)$ is the Fourier transform of the impulse transient function $h(t-\tau) . H(\omega)$ characterises the frequency properties of the measuring equipment. Then,

$u(\omega)=u_{T}(\omega)+\frac{v(\omega)}{H(\omega)}$

where $u_{T}(\omega)$ is the Fourier transform of the accurate measured signal.

The application of the inverse Fourier transform to equation (22) gives the formula

$u(t)=u_{T}(t)+\frac{1}{2 \pi} \int_{-\infty}^{+\infty} \frac{v(\omega)}{H(\omega)} e^{-j \omega t} d \omega$,

in which the integral diverges, as a rule. The reason for that is the presence of high frequencies in the function $v(\omega)$ and the excess of the degree of a denominator under the degree of a numerator in $H(\omega)$. The idea of the regularisation method is the suppression of the influence of high frequencies $\omega$, multiplying the function $\frac{y(\omega)}{H(\omega)}$ by a stabilising factor $f(\omega, \alpha)$, which depends on the parameter $\alpha$ and frequency $\omega$. A. N. Tikhonov [22] proposes determining the stabilising factor as

$f(\omega, \alpha)=\frac{|H(\omega)|^{2}}{|H(\omega)|^{2}+\alpha M(\omega)}$

where $M(\omega)$ is a given even function of $\omega$. With this in mind, we obtain the classes of the regularised solutions of integral equation (12): 
$u_{\alpha}(t)=\frac{1}{2 \pi} \int_{-\infty}^{+\infty} \frac{H(-\omega) \cdot y(\omega)}{H(\omega) \cdot H(-\omega)+\alpha \cdot M(\omega)} e^{-j \omega t} d \omega$.

In this case, one of the possible variants of the polynomial $M(\omega)$ takes the form

$M(\omega)=\sum_{n=0}^{p} q_{n} \omega^{2 n}$

where $q_{n}$ is a given non-negative constant.

It is suggested that the value of parameter $\alpha$ should be found according to 'discrepancy'. Let us identify the limiting error $\delta$ of determining the output signal of the measuring equipment $y(t)$. Let us define the 'discrepancy' of the approximate solution:

$\rho(\alpha)=\int_{-\infty}^{+\infty}\left[\int_{o}^{t} h(t-\tau) u_{\alpha}(\tau) d \tau-y(t)\right]^{2} d t$.

We choose a monotonic sequence of numbers $\alpha_{0}, \alpha_{1}, \ldots, \alpha_{n}$, e.g. a geometric progression. For each value of $\alpha_{i}$, the 'discrepancy' $\rho\left(\alpha_{i}\right)$ is calculated. As the desired value of $\alpha$, we take such $\alpha_{k}$, for which the equality $\rho\left(\alpha_{k}\right)=\delta$ is fulfilled with the required accuracy. The approximate solution of this equation can be found with the help of other numerical methods; for example, Newton methods [23], [24].

In [24], an optimal regularised solution of integral equation (12) is obtained when the additional information on the spectral densities of the output noise of the measuring equipment $S_{v}(\omega)$ and the input signal $U(\omega)$ is available. This solution does not depend on parameter $\alpha \alpha$ and has the form

$$
U_{o p t}(t)=\frac{1}{2 \pi} \int_{-\infty}^{+\infty} \frac{H(-\omega) \cdot y(\omega)}{H(\omega) \cdot H(-\omega)+\frac{S_{v}(\omega)}{U(\omega)}} \cdot e^{-j \omega t} d \omega .
$$

This result coincides with the result of applying the optimum filtration according to Wiener [25], [26].

In many practical cases, the spectral density of noise of the output signal $y(t)$ can be determined according to the observation results. It is more difficult to specify the spectral density of the input signal $u(t)$. Its evaluation can be obtained on the basis of a priori information about the measured signal. This complicates the task of the optimal restoration of the input signal of the measuring equipment.

The impulse transient function used above is not the only characteristic that reflects the dynamic properties of the measuring equipment. The dynamic properties can also be defined by a differential equation with the corresponding coefficients; a transfer function; and a set of amplitudefrequency and phase-frequency characteristics [5]. The use of

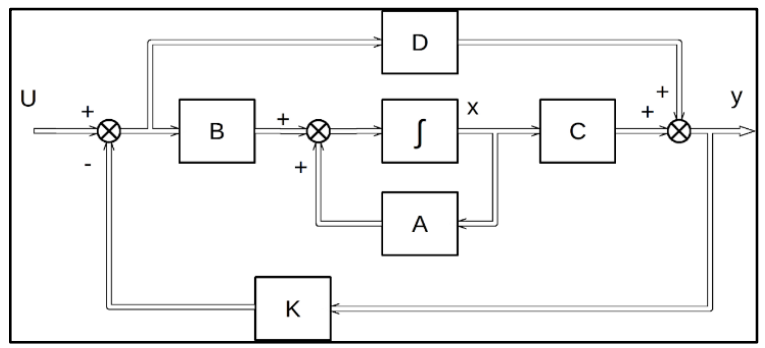

Figure 13. Structural diagram of the automatic control system.

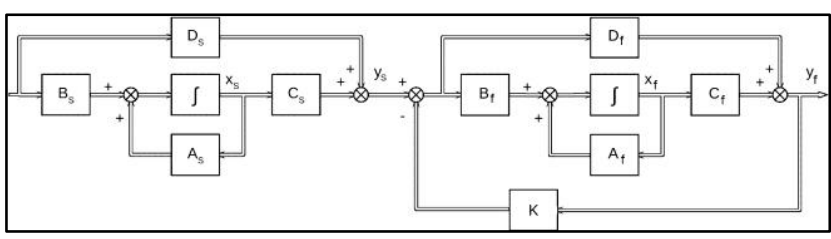

Figure 14. Structural diagram of the dynamic measuring system.

these characteristics enables the development of relevant methods for the restoration of a dynamically distorted signal or to correct dynamic errors.

The use of differential equations enables the development of methods of dynamic measurements based on the results of automatic control theory. [27] presents a review of automatic control theory and the prospects of its application in measurement technology. In [28], the term 'dynamics' is considered from different angles in respect to the problems of metrology. The importance of studying measuring signals is noted. A classic description of dynamic systems on the basis of differential equations is given.

The author pays attention to the related concepts of the properties and behaviours of systems. The concepts of the stability, stationary state, and drift of dynamic systems are also analysed in the work. The basis for applying the methods of automatic control theory to the correction of dynamic measuring errors is the similarity of structures of automatic control systems and dynamic measuring systems [32]. The classic structure of the automatic control system is illustrated in Figure 13, which is a graphical representation of the system of differential equations.

In Figure 13, $u$ is an input signal of the system; $x$ is a state vector of the system; $y$ is an observation vector; and $A, B, C, D$ are the corresponding matrices. The required dynamic properties are provided by setting the coefficients of the feedback matrix $K$.

In dynamic measuring systems, it is impossible to establish the feedback from the output to the input, since the input's measured action is not available. That is why, in such systems, the possibility of reducing measurement errors is realised by means of a special filter at the output of a primary measuring transducer (sensor). In this case, the structural diagram of the dynamic measuring system is shown in Figure 14.

In Figure 14, $\mathrm{u}$ is an input measured vector of the system; $x_{S}, y_{S}$ are state and observation vectors; $A_{S}, B_{S}, C_{S}, D_{S}$ are corresponding matrices of the sensor; $x_{f}, y_{f}$ are vectors of the state and observation of the filter; $A_{f}, B_{f}, C_{f}, D_{f}$ are filter matrices of the corresponding dimension; and $K$ is a matrix for setting the coefficients to provide the required dynamic error. In this system, based on the similarity of the sensor and filter structures, the latter can be considered as a dynamic sensor model. Therefore, dynamic measuring systems have been developed based on the models for creating automatic control systems outlined in [30].

A dynamic measuring system with modal control of dynamic characteristics has the following structure, as illustrated in Figure 15

At this time, the transfer functions of sensor $W_{S}$ and a model of sensor $W_{M S}$ are equal. If the output signals $y_{S}$ and $y_{M S}$ are close to each other, then the input signals $u$ and $u_{M S}$ will differ from each other slightly.

Consequently, the input signal of the model $u_{M S}$ can be used to judge the input signal of the sensor $u$. This is the basic idea 


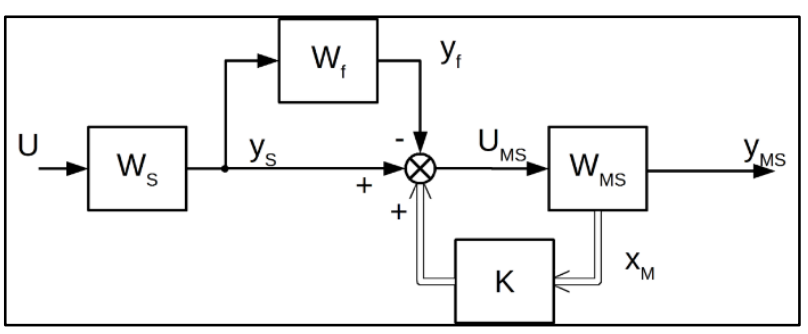

Figure 15. Structural diagram of a dynamic measuring system with modal control of dynamic characteristics.

of the correcting the dynamic measuring error. In this system, the transfer function $W_{D S}(p)=u_{M S}(p) / u(p)$ can be obtained with any given zero and poles. If

$W_{S}(p)=\frac{b_{m} p^{m}+b_{m-1} p^{m-1}+\cdots+b_{1} p+b_{0}}{p^{n}+a_{n-1} p^{n-1}+\cdots+a_{1} p+a_{0}}$,

then the transfer function of the dynamic measuring system can be obtained by

$W_{D S}(p)=\frac{\left(b_{m}-d_{m}\right) p^{m}+\left(b_{m-1}-d_{m-1}\right) p^{m-1}+\cdots+\left(b_{0}-d_{0}\right)}{p^{n}+\left(a_{n-1}-K_{n-1}\right) p^{n-1}+\cdots+\left(a_{1}-K_{1}\right) p+\left(a_{0}-K_{0}\right)}$

where $d_{0}, d_{1}, \ldots, d_{m}$ are configurable filter parameters $W_{f}$, and $K_{0}, K_{1}, \ldots, K_{n-1}$ are configurable feedback vector parameters of $K$ [31].

The development of this method for the observed state coordinate vector is outlined in [31], [34]. If the spectral density of the measuring signal $u$, and the observed coordinates are known, then the structural diagram of this measuring system is shown in Figure 16.

If the spectral densities of the measuring signal $S_{u}(\omega)$ and noises $S_{v_{2}^{1}}(\omega), \ldots, S_{v_{2}^{m}}(\omega)$ in the channels of the observed state coordinates are known, then, in [33], the optimum individual characteristics of the correcting device that reduces the measuring error are obtained.

Dynamic measuring systems based on the iterative principle of the measuring signal recovery are given in [32], [34]. In the structure of such a measuring system, there are several sensor models. Every subsequent model processes the measuring errors of the previous ones. The main property of such measuring systems is their low sensitivity to sensor noise and their ability to reduce dynamic error without the significant increase of the system bandwidth.

The structural diagram of this measuring system is shown in Figure 17 [29].

In [32], [35], dynamic measuring systems in which a sliding mode is implemented are observed and explained. The structures ensuring the stability of such systems are analysed. A combination of high dynamic accuracy and low sensitivity to sensor noise is shown. A structural diagram of a dynamic measuring system in sliding mode is given in Figure 18.

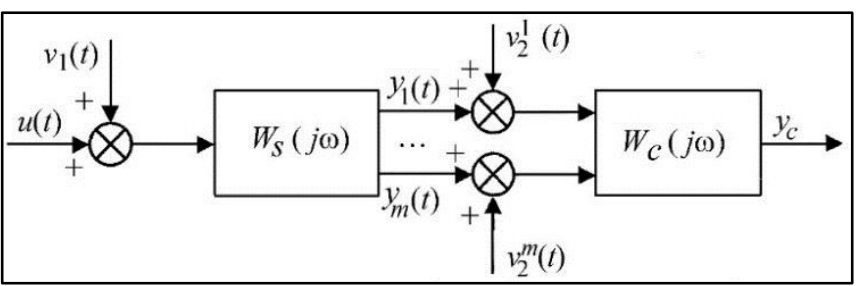

Figure 16. Structural diagram of a measuring system with the observed state coordinate vector.

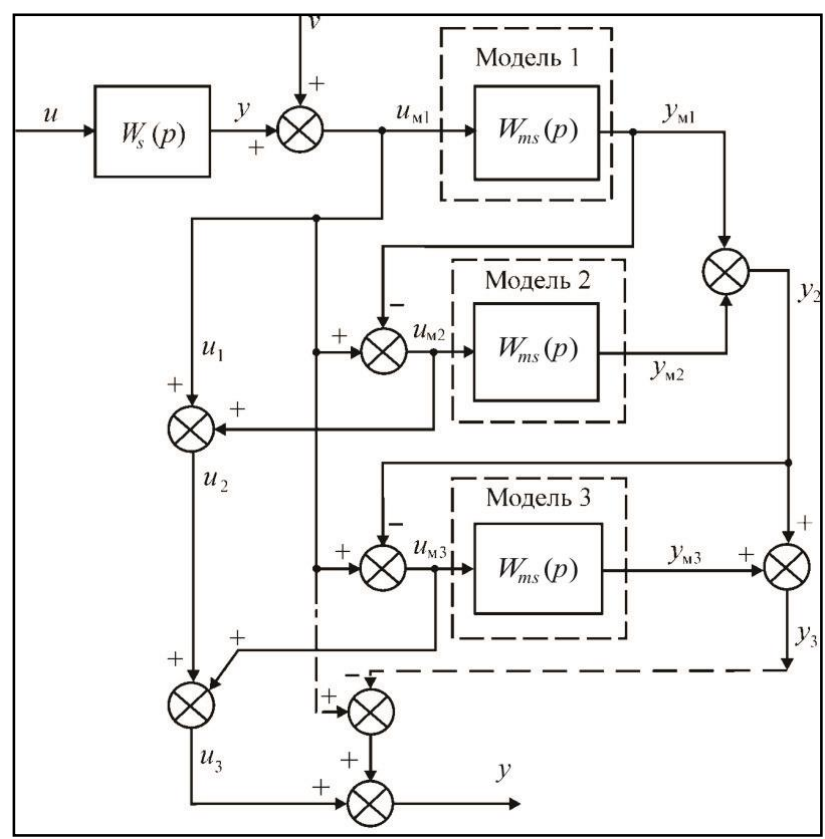

Figure 17. Structural diagram of an iterative dynamic measuring system.

The presence of a relay element for a high-order system may cause instability of the system. To ensure stability a cascading structure of the measuring system is given in reference [32]. Every subsequent cascade restores the measuring signal more accurately than the previous one. The results of the system modelling in a sliding mode with three cascades are shown in Figure 19.

In [32], [36], dynamic measuring systems are observed on the basis of neural network structures. The stability conditions of such systems are investigated therein. Special means of ensuring the suppression of sensor noise are introduced to the system structures. The unique feature of neural network dynamic measuring systems is their ability to adapt to the type of such measuring signals and to reduce the measuring error in the multiple measuring of such signals.

A feature of dynamic measuring systems developed on the basis of methods of automatic control theory is the possibility to obtain an additional channel for dynamic error estimation [32]. An error in an estimate of a dynamic error is comparable with a measuring error of the input signal of the measuring system.

A structural diagram of the channel for dynamic error estimation is shown in Figure 20.

In [32], [37], intelligent dynamic measuring systems that can adapt their parameters to dynamic measuring errors are

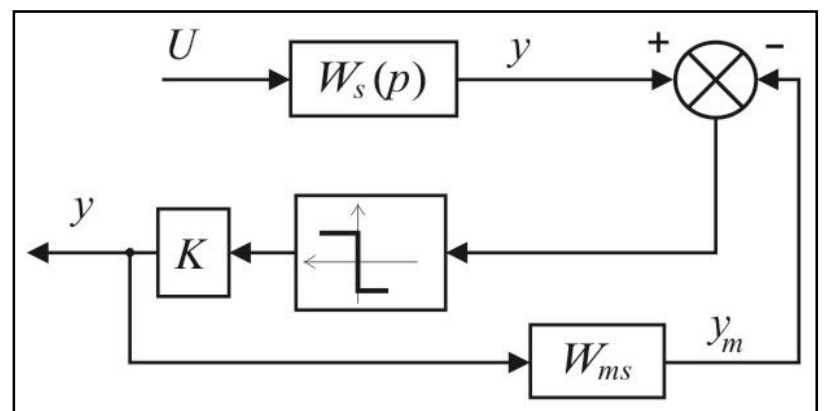

Figure 18. Structural diagram of a dynamic measuring system in sliding mode. 


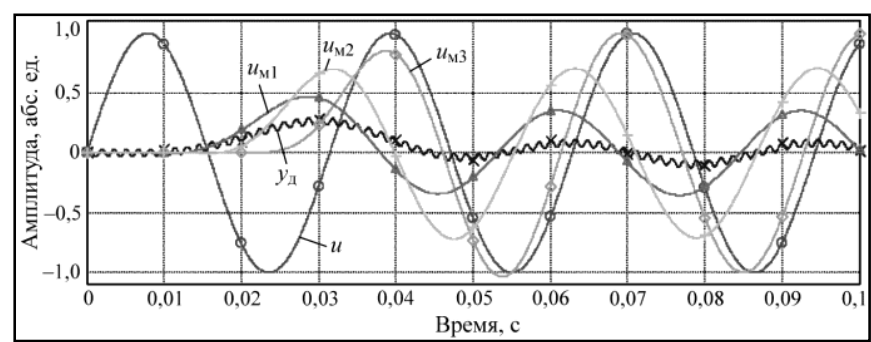

Figure 19. Results of modelling a tri-cascade dynamic measuring system in sliding mode.

considered. Measuring systems with parameter adaptation after the performance of measurements during the processing of their results are considered. Measuring systems with adaptation of dynamic parameters in the process of measuring are also given.

Based on ideas of automatic control theory, [38] considers dynamic measuring systems from the standpoint of simultaneous measurement and observation. A structural diagram of this measuring system is shown in Figure 21. Here, $P$ is a dynamic process in the system; $M U$ and $M Y$ are measurement processes $u(t)$ and $y(t)$ correspondingly; and $x_{\text {obs }}(t)$ is the observation process.

The use of the observation process allows us to refine the model of the process P. This approach is mainly focused on multi-connected dynamic measuring systems, where $u$ is the vector of the measured coordinates. Its implementation is based on the use of the methods and results of automatic control theory, which are well developed at present [39]. This approach is promising both from the standpoint of the development of the general theory of dynamic measurements and in applications to dynamic measurements of the specific systems.

Another approach in dynamic measurements that considers the uncertainty of the dynamic model is based on the use of chaos theory. Thus, in [40], compensation for the dynamic error is considered in the presence of uncertainty in the coefficients of the sensor dynamic model. The uncertainty of these coefficients is expressed on the basis of the method of polynomial chaos theory. The method also assumes the description of the input measured signal in an analytical way. This is its essential limitation. This method gives an estimate of the measurement error and takes into account noise at the sensor output.

\section{IDENTIFICATION OF THE DYNAMIC CHARACTERISTICS OF A MEASURING EQUIPMENT}

Concerning the identification of dynamic characteristics and methods of solving this problem, measuring equipment does not differ from automatic control systems. For automatic control systems, a great many identification methods have been

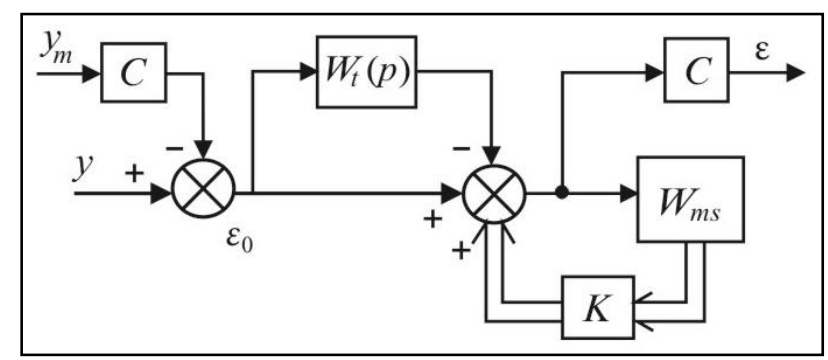

Figure 20. Structural diagram of a channel for dynamic error estimation.

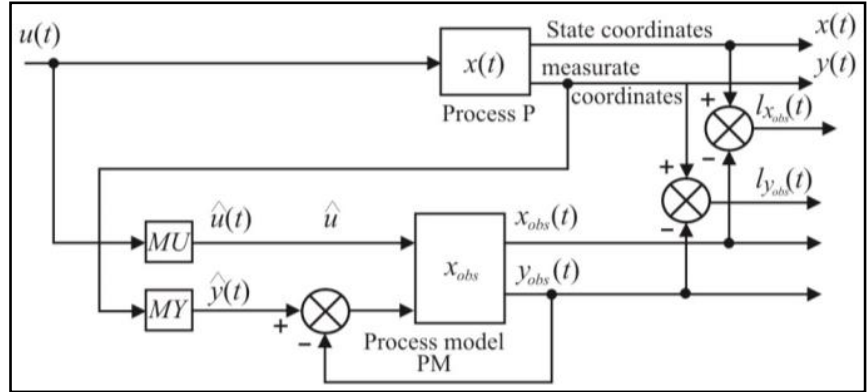

Figure 21. Structural diagram of the measuring process and observation.

developed [41]. However, these methods do not pay much attention to the problem of accuracy in the identification of dynamic characteristics. However, they are an important feature of measuring equipment. The approach of determining dynamic characteristics with an estimate of their accuracy was proposed by V. A. Granovskii [5]. The determination of dynamic characteristics requires the formation of test signals (Figure 22).

On a number of test signals, he identified errors in the determination of the transient characteristics, impulse response, and frequency characteristics. He proposed a method for the multiple integration of differential equations of the measuring equipment, which helps to determine the coefficients of differential equations and errors in their determination for the input test signals of an arbitrary shape. He developed an adaptive method for the identification of dynamic characteristics. This method determines the transfer function by the subsequent complication of the transfer function, step by step, and identifies optimal coefficients at every step. Based on the minimisation of integral quadratic error, this method makes use of five types of test signals: stepwise; exponential; and three impulse signals of triangular, cosine, and sinusoidal forms.

The determination of impulse and transfer functions by means of statistical regularity is outlined in [42], [43]. In fact, determining the dynamic characteristics of measuring equipment is not an easy task, especially for multi-axis sensors and multichannel measuring systems. Quite often, the test equipment is a complicated facility. In the vast majority of cases, the specification of requirements for the test equipment is made by means of the expert method, and the accuracy of the identification of dynamic characteristics is not estimated. There are several specific examples of the determination of dynamic characteristics, given below.

In [44], the technology for the determination of dynamic characteristics for a six-component force-and-moment sensor is considered. Eigen frequencies for each of the six coordinates are considered to be such characteristics. The study offers two methods of obtaining these frequencies. The computational method uses an ANSYS SOFTWARE simulation. The experimental method measures the frequency of natural oscillations after impulse force.

In [45], the method used for the dynamic calibration of the force measurement devices is considered. In this case, a

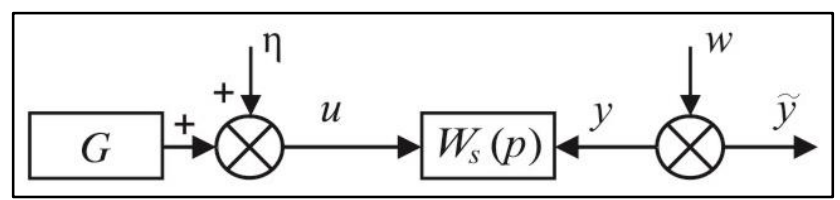

Figure 22. Structural diagram of the equipment used to identify dynamic characteristics. 
standard force dynamometer and force dynamometer to be tested connected in a series through the load frame in the general structure are used. By exciting the oscillations of this structure, the frequency response of the force measuring device is defined, including the natural oscillation frequency.

In [46], the experimental determination of the dynamic characteristics of the mechanical system is considered. Based on measurements with accelerometers and interferometers, the amplitude-frequency and phase-frequency characteristics of the system along the axes are determined. Frequency characteristics of noise in a mechanical system are also estimated.

In [47], the influence of a dynamic effect on the process and accuracy of a flowmeter calibration is considered. The dynamic properties of flowmeters are given in the form of a delay in the signal for converting the measuring flow signal into an electrical signal. The study proposes that the model of dynamics is described by the equation of the first order. Its parameters are determined in the process of calibration. This reduces the measurement uncertainty of the flowmeter.

In [48], the calibration method of a torque measuring transducer operating in dynamic modes is considered. This method is based on an accurate estimate of mass angular acceleration with a known momentum of inertia.

The researchers in [49], [50] consider the determination of the frequency characteristics of the kernel of the Volterra equations for single- and multi-channel dynamic systems. This method is based on the use of the Fourier transform when applying known harmonic (sinusoidal) signals to the input of the dynamic system. This method identifies the frequency characteristics of a dynamic system with non-linearities. This method is pointed to the application in dynamic measuring systems to measure the electrical signals in a broad band.

The method of dynamic calibration of a high-pressure sensor based on a quasi- $\delta$ function is considered in [51]. This signal is given in the form of a half sine wave with the duration of several microseconds, $\mu$ s. Using the fast Fourier transform of the input quasi $\delta$-signal $f(t)$ and the output signal $y(t)$ :

$F(\omega)=\mathrm{fft}[f(t)], Y(\omega)=\mathrm{fft}[y(t)]$

and the normalised frequency response of a pressure sensor is determined experimentally

$H(\omega)=\left|\frac{\frac{Y(\omega)}{Y(0)}}{\frac{F(\omega)}{F(0)}}\right|$.

The quasi $\delta$-impulse is created by the bullet going through the Hopkinson bar.

The researchers in [52] consider the behaviour of a dynamic measuring system in the neighbourhood of a user-specified frequency. A method of approximation of a frequency response in the neighbourhood of the frequency by polynomials is proposed. The polynomial approximation takes into account the non-zero initial conditions of a dynamic system. However, in this case, it does not take into account disturbances and noise in the system, considering that at frequency response approximation, the input and output signals of the measuring system are known. The error of the polynomial approximation is also studied. This method is pointed to the analysis of the behaviour of a dynamic measuring system concerning the measurement of harmonic (sinusoidal) signals in the neighbourhood of a certain frequency.
The creation of mathematical models of measuring equipment that adequately reflects the properties has made it possible to develop measuring equipment with optimal characteristics and to obtain higher measurement accuracy when processing measuring results. This approach is implemented in mathematical models, and software is created in NPL. Thus, in [53], one can see the application of the method of mathematical simulation to analyse dynamic measurements executed in NPL. The first example demonstrates the mathematical model of a lock-in amplifier. The comparison between the operation of a lock-in amplifier in a real experiment and the results of the simulation reveals sources of additional error. The second example considers simulation of an underwater acoustics network of three hydrophones. The case is considered when one of the sensors has a smaller bandwidth than the others. The software helps to simulate the operation of this network under various conditions. The third example considers an imperfect clock with a quartz oscillator. The accuracy of the temporal data fit has a direct influence on dynamic error. Based on the Monte Carlo method, this software is used in the simulation of the long-term behaviour of the timing device, taking into account long-term drift and jitter within the measurement data. The fourth example presents a shock tube as a means of generation of calibration pressure signals with a broad bandwidth. The effects in a shock tube are simulated on the basis of wave propagation in a shock tube. The simulation enables the determination of the optimal parameters of a shock tube.

[81] is devoted to the development and application of a new open-source software package for the analysis of dynamic measurements.

At the same time, in many publications, the question of the determination of dynamic characteristics and measurements in different problems is analysed.

In [54], [55], the method of autoregressive analysis of the spectrum of the output signal is used to determine the dynamic characteristics of the pressure transducer (the transfer function in the frequency domain and the transient function in the time domain). In this case, the spectral analysis is undertaken on the basis of the Fourier transform of the transducer response to a step change in the pressure signal. The results of the experimental studies show good correspondence between the characteristics of the obtained dynamic models and real transducers.

A generator of dynamic pressure with a rotating valve is proposed in [56]. The methods of frequency response estimation and the speed of the pressure transducer are developed on the basis of an analysis of the transducer's reaction to the generated signal as a series of rectangular pressure impulses with variable frequencies and amplitudes. Experimental studies have shown good repeatability of the amplitude spectrum of the generated signal and the possibility to compare the frequency responses of two pressure transducers while simultaneously observing their output signals.

In [57], a vibration pressure transducer with a frequency output is considered as the object of analysis. To determine the dynamic characteristics of a transducer, the method of parametric identification is used with a harmonic input action and a controlled average value and amplitude. When modelling the measuring system, the method of computational hydrodynamics is used. The results of the modelling allow us to reveal the effects during the operation of the given pressure transducer, which influence dynamic characteristics a great deal. 
In [58]-[64], methods of developing the variable effects of different types of dynamic calibration of pressure transducers are considered. In [58] and [59], generators of impulse pressure in the form of a short sinusoid half-wave are presented. The principle of operation of the first thereof is the use of the system with drop mass, and the second is based on the use of the system with a Hopkinson bar and a piston gauge. In [60] and [61], the system of calibration with the generator of a periodically varying pressure in the form of a double-acting pneumatic actuator is considered. Methods of dynamic calibration that are based on the use of a shock tube as a generator of step-change pressure are studied in [62]-[64]. Dynamic calibration with the use of one or another calibration action involves the determination of the dynamic characteristics of the resulting impulsive, amplitude-frequency, or transient functions of pressure transducers.

An experimental method of the determination frequency response of a pressure sensor of the second order is considered in [64]. The experimental installation generates the input step signal. According to the transient process, the parameters of transfer function are determined, and the frequency characteristics are determined from them.

The methods used for the dynamic calibration of different types of conditioning amplifiers are presented in [82].

The necessity for the dynamic measurement of pressure arises at low [65] and high [66] temperatures of gases and liquids [67].

There are also examples of the use of the Kalman filter to determine the parameters of a dynamic measuring system.

The researchers in [68] consider the method of dynamic weighing with letter scales. For the model of scales of the second order, the coefficients of the difference equations of weights and the weight of an object are determined with the Kalman filter.

The solution to dynamic measurement problems in specific applications is characterised by the great variety thereof and particular methods of resolving them. The correction of a dynamic error is undertaken on the basis of setting acceptable dynamic properties for a specific problem.

[69] considers the correction of a dynamic error of an auxiliary wale heat flux sensor. This sensor is described by the differential equation of the first or second order. The correction of a dynamic error is performed by the inverse transfer function, the parameters of which are selected according to an acceptable dynamic error.

There are studies wherein analysis of the dynamic properties of measuring equipment helps to develop the optimal structure of the measuring equipment.

There are studies that found that the dynamic measuring equipment has nonlinearities.

In [70], an approach to develop magnetic-field sensors using a nonlinear dynamic equation describing the operation of the sensors is considered. The basic dynamic equation is the following:

$$
\tau \frac{d x}{d t}=-x+\tanh \left[\frac{x+h(t)}{T}\right]
$$

where $x$ is the strength of a magnetic field; $h(t)$ is an external magnetic field; $\tau$ is the system time constant; and $T$ is the temperature.

On the basis of an analysis of this equation (2), states of the sensor having minimum potential energy are identified. This essentially nonlinear differential equation is used to create an optimal sensor circuit.

In [71], dynamic measurements of the composition of gases in chemical industry are considered. Chemical sensors are characterised by inertia and have nonlinear statistical characteristics. The following inverse model of a dynamic measuring system is given.

$u[n-k]=\sum_{j=1}^{n} B_{j}(q) y[n]+c[n]$,

where $B_{j}(q)$ is a polynomial in the delay operator $q(q u[n]=$ $u[n-1]), u[n]$ and $y[n]$ are output and input signals of the inverse system, respectively, and $c[n]$ is a residual term. The parameters of the model are determined by minimising the measuring error in the test experiments.

The studies on this topic also give information about completely unexpected effects in dynamic measurements. In [72], four algorithms for the compensation for static measurement error in the operation of the measuring device in dynamic mode are considered. These algorithms are based on the linear statistical characteristics of the measuring device, with the following methods: the piecewise linear interpolation compensation algorithm, polynomial progressive compensation algorithm, improved polynomial progressive compensation algorithm, and artificial neural network compensation algorithm. There are no parameters of dynamic characteristics in the algorithms. Testing them in dynamic mode has shown that they have quite certain frequency characteristics. In this case, the bandwidth of the first three algorithms is approximately the same, but the bandwidth of a neural algorithm is slightly larger.

[73] considers the system of pipelines in the form of a set of concentrated masses described by the system of differential equations. The problem of determining the frequencies of the oscillations of the system is solved by the method of the theory of inverse problems solution, with boundary conditions at the ends of the pipeline.

[74] is devoted to the use of the Bayesian estimation problem to measure spatial coordinates of the objects. Here, in the general case, a discrete variant of a nonlinear dynamic system is considered, in which a coordinate vector of the state to be measured is connected with a vector of the observed coordinates by the known model. The technique used for computing spatial coordinates is traditional and requires the knowledge of their probability density function and noise probability densities.

[75] studies the rheological properties of drops by means of experimental installation with a capillary, piezo translator, and pressure sensor. A particular characteristic of the experimental installation is obtained. The results of the research are obtained on the basis of the Fourier transform of pressure sensor signals accounting for the particular characteristic of the experimental installation.

In [76], the properties of thermal electric transducers are studied by measuring their electrical resistance at various frequencies. The dynamic model of an integrated electrical resistance is given. The natural frequencies of this model's poles provide information on the thermal electrical transducer. Although the problem of restoring a dynamically distorted signal or correcting a dynamic progression is not solved here, the studies conducted are based on an analysis of the dynamic properties of the object. 
The researchers in [77] propose a method of estimation of square error criterion for measuring the signals by an accelerometer with a dynamic model of the second order. It is shown therein that the maximum error occurs when measuring triangular and trapezoidal signals. For measuring signals of different forms, the methods of their approximation are given on the basis of a genetic algorithm to obtain the maximum quadratic measuring error of these signals. The authors suggest using this method to calibrate accelerometers in dynamic measurement.

[78] considers the design of a dynamic compensator for a second-order temperature sensor. The compensator parameters of the second order are determined by the Kalman filter adapted to evaluate the parameters of a dynamic compensator.

The problem of reducing a dynamic error in the measurement of forces by the tri-component dynamometer of a micro-machine is considered in [79]. At the initial stage, the matrix of the frequency characteristics of the tri-component dynamometer is identified accounting for the cross links on the measured coordinates. Compensation for a dynamic error is performed on the basis of multiplying the observed force vector by the inverse matrix of the frequency response. In the article, the problem of the regularity of the inverse matrix and suppression of the influence of high-frequency noise when it is reversed is not discussed.

[80] considers a dynamic model of a sensor to measure the convective heat transfer coefficient of buildings. This dynamic model is presented by a differential equation of the third order. On the basis of the developed model, the design parameters of the sensor, ensuring its higher dynamic characteristics, are selected. The dynamic measuring error is determined by a simple operation of multiplication time interval by the rate of change in the transfer of convective energy.

\section{CONCLUSION}

In conclusion, it is important to note that this report does not fully cover the topic of dynamic measurements. The author has simply attempted to discuss the basic results of the theory of dynamic measurements and to give examples of several of their specific applications. The number of the examples presented in the articles is large. The author has been able to analyse only a small part of them. At present, the author feels the growing interest in such measurements. One of the objective reasons for this growing interest is the necessity to create new technology.

\section{REFERENCES}

[1] A. Cornu, Sur la condition de stabilite du movement d'un systeme oscilant soumis a une liaison synchrinique pendulaire, C.r. Acad. sci. 104, 22 (1887) p. 1463 (in French).

[2] A. Blondel, Oscillographes, nouveaux apparelis pour l'etude des oscillations electriques lentes, C.r. Acad. Sci. 116, 10 (1893) p. 502 (in French).

[3] D. I. Mendeleev, About methods of precise or metrological weighing, in: Papers on Metrology, VNIIM, Standart gid, Moscow, 1936, pp. 67-164.

[4] A. N. Krylov, Some notes about crushers and indicators, in: Izvestia Saint Petersburg A. N., 1909, pp. 623-655.

[5] V. A. Granovsky, Dynamical measurements: fundamentals of metrology software, Energoizdat, Leningrad, 1984 (in Russian).

[6] A. L. Shestakov, Analysis of dynamic error and selection of a measuring transducer's parameters at stepwise, linear and hierarchic segments, Izmeritelnaya tekhnika [Measuring Engineering] 6, 13 (1992).

[7] E. Layer, W. Gawedzki, Theoretical principles for dynamic errors measurement, Measurement 8, 1 (1990), pp. 45-48.

[8] E. Layer, Mapping Error of linear Dynamic systems caused by reduced-order model, IEEE Transactions on Instrumentation and Measurement 50, 3 (2001) p. 792.

[9] E. Layer, Non-standard input signals for the calibration and optimization of the measuring systems, Measurement 34 (2003) p. 179.

[10] K. Tomezyk, E. Layer, Energy density for signals maximizing the integral-square error, Measurement 90 (2016) p. 224.

[11] E. Layer, Theoretical principles for establishing a hierarchy of dynamic accuracy with the integral-square-error as an example, IEEE Transactions on Instrumentation and Measurement 46, 5 (1997) p. 1178

[12] V. V. Solodovnikov, Statistical dynamics of linear systems of automated control, Fizmatgiz, Moscow, 1960.

[13] H. James, N. Nichols, R. Phillips, Theory of Servomechanisms (I.L., 1951).

[14] A. F. Verlan, V. S. Sizikov, Integral Equations: Methods, Algorithms, and Programmes (Resource Book) Naukova dumka, Kiev, 1986.

[15] V. I. Krylov, Approximate calculation of integrals (M.: Nauka, 1967).

[16] V. I. Krylov, L. T. Shulgina, Reference book on numerical integration (M.: Nauka, 1966).

[17] G. I. Vasilenko, Signals renewal theory: from reduction to the ideal device in physics and technique (M.: Sov.radio, 1979).

[18] S. G. Rautian, Real spectral apparatus, Sov. Phys. Usp 66, 3 (1958) p. 27.

[19] E. I. Buzz, Sharpening of observational data in two dimensions, Australian J. of Phys. 7, 1 (1955) p. 67.

[20] G. N. Solopchenko, Ill-posed problems of measuring engineering, Izmeritelnaya tekhnika [Measuring Engineering] 1, 51 (1974).

[21] K. Hein, A. Lesniewski, Digital correction of the dynamic error arising in the input circuits of measuring instruments, Measurement 2, 4 (1984) p. 170.

[22] A. N. Tikhonov, V. Ya. Arsenin, Solutions of ill-posed problems (M.: Nauka, 1979).

[23] V. A. Morozov, On regularization methods for ill-posed problems and regularization parameter selection, DANSA 6, 1 (1967) p. 43.

[24] V. A. Morozov, On the solution of functional equations by the method of regularization, DANSA 6 (1967) p. 175.

[25] N. Wiener, Extrapolation, interpolation and smoothing of stationary time series with engineering applications, John Wiley, New York, 1950.

[26] S. Twomey, The application of numerical filtering of the solution of integral equations encountered in indirect sensing measurements (J. Franklin Inst., 1965).

[27] S. Engelberg, Control theory, part 1, IEEE Instrumentation and Measurement Magazine 11, 3 (2008) p. 34.

[28] K. H. Ruhm, Dynamic and stability: A proposal for related terms in metrology from a mathematical point of view, Measurement 79 (2016) p. 276

[29] A. L. Shestakov, Measuring transducer of the dynamic parameters with the iterative principle for signal restoration, Devices and systems. Management, control, diagnostics 10 (1992) p. 23.

[30] B. N. Petrov, V. Yu. Rutkovskii, I. N. Krutova, Construction principles of self-adjusting control system, Mashinostroenie, Moscow, 1972.

[31] A. L. Shestakov, Dynamic error correction method, IEEE Transactions on Instrumentation and Measurement 45, 1 (1996) p. 250.

[32] A. L. Shestakov, The automatic control theory methods in dynamic measurements (Chelyabinsk, South Ural State University, 2013) (in Russian). 
[33] A. L. Shestakov, D. Yu. Iosifov, Zeros and transfer function poles control of measuring converter with measurable sensor status vector, SUSU Bulletin, Computer Science, Management, Electronic Engineering Series, 4, 2 (2003) p. 42.

[34] A. L. Shestakov, Detector element of dynamic parameters with signal restriction iteration principles, Apparatus and Control Systems, 10 (1992) p. 23.

[35] A. L. Shestakov, M. N. Bizyaev, Dynamically distorted signals recovery of testing measurement system by sliding mode approach, Proc. of RAS. Energetics, 6, 119 (2004), pp. 119-130.

[36] A. S. Volosnikov, A. L. Shestakov, Neural network dynamic model of measurement system with regenerate signal filtration SUSU Bulletin, Computer Science, Management, Radio Engineering Series, 14, 69 (2006), p. 16-20.

[37] A. L. Shestakov, E. V. Soldatkina, Measuring Transducer With Parameters Self-Adjusted by Dynamic Error Criterion in Information-Measuring and Operated Systems and Devices: Subject Collection of Research Papers, SUSU Publishing, Chelyabinsk, 2000, pp. 126-134.

[38] K. H. Ruhm, Measurement plus observation: A new structure in metrology, Measurement 126 (2018) 421-432,

DOI: https://doi.org/10.1016/i.measurement.2017.03.040

[39] A. T. Ruban, Adaptive Control with Identification, Tomsk University Publishing, Tomsk, 1983.

[40] A. Monti, F. Ponci, Uncertainty evaluation under dynamic conditions using polynominal chaos theory, IEEE Transactions on Instrumentation and Measurement 59, 11 (2010) p. 2825

[41] P. Eikhof, System Identification, John Wiley, London, 1974.

[42] G. N. Solopchenko, Method of statistical regularization, Proc. of VELNII, 5, 43 (1970).

[43] N. Kh. Ibragimova, G. N. Solopchenko, Assessment of the weighing and transfer function using statistical regularization, Proc. of VELNII, 16, 86 (1973).

[44] L. Ying-jun, G.-C. Wang, Y. Zhang, et al., Dynamic characteristics of piezoelectric six-dimensional heavy force/moment sensor for large-load robotic manipulator, Measurement 45, 5 (2012) p. 1114.

[45] R. Kumme, Investigation of the comparison method for the dynamic calibration of force transducers, Measurement 23, 4 (1998) p. 239.

[46] R. S. Michelini, G. B. Rossi, Computerized test scheme for the motion recognition and evaluation of a dynamic system, Measurement 5, 2 (1987) p. 61.

[47] R. Engel, H.-J. Baade, Qualifying impacts on the measurement uncertainty in flow calibration arising from dynamic flow effects, Flow Measurement and Instrumentation 44 (2015) p. 51.

[48] R. S. Oliveira, S. Winter, H. A. Lepikson, et al., A new approach to test torque transducers under dynamic reference regime, Measurement 58 (2014) p. 3554.

[49] M. Schetzen, The Volterra and Wiener Theories of Nonlinear Systems, John Wiley, New York, 1980.

[50] M. Alizadeh, S. Amin, D. Ronnow, Measurement and analysis of frequency-domain Volterra kernels of nonlinear dynamic 3x3 MIMO systems, IEEE Transactions on Instrumentation and Measurement 66, 7 (2017) p. 1893.

[51] Y. Zhang, J. Zu, H.-Y. Zhang, Dynamic calibration method of high-pressure transducer based on quasi- $\delta$ function excitation source, Measurement 45, 8 (2012) p. 1981.

[52] J. Schoukens, G. Vandersteen, R. Pinelon, et al., Bounding the polynomial approximation errors of frequency response functions, IEEE Transactions on Instrumentation and Measurement 62, 5 (2013) p. 1346.

[53] T. J. Esward, Investigation of dynamic measurement applications through modeling and simulation, Technisches Messen 83, 10 (2016) p. 557.

[54] R. Sanchis, I. M. Tkachenko, G. Verdu, et al., Dynamic analysis of the NPP pressure sensor system, Progress in Nuclear Energy 29, 3-4 (1995) p. 321.
[55] H. Chang, P. K. Tzenog, Analysis of the dynamic characteristics of pressure sensors using ARX system identification, Sensors and Actuators A: Physical 141, 2 (2008) p. 367.

[56] T. Kobata, A. Ooiwa, Method of evaluating frequency characteristics of pressure transducers using newly developed dynamic pressure generator, Sensors and Actuators A: Physical 79, 2 (2000) p. 97.

[57] H. Zhuang, S. C. Fan, Z. S. Guo, D. Z. Zheng, Dynamic characteristics analysis of vibrating cylinder pressure transducers (VCPT), Sensors and Actuators A: Physical 157, 2 (2010) p. 219.

[58] Y. Durgut, E. Aksahin, E. Batci, et al., Preliminary dynamic pressure measurement system at UME, in Proc. of the $21^{\text {st }}$ IMEKO World Congress 30 Aug.- 4 Sept. 2015, Prague, Czech Republic, pp. 181-184.

[59] Y. Zhang, J. Zu, H.-Y. Zhang, Dynamic calibration method of high-pressure transducer based on quasi- $\delta$ function excitation source, Measurement 45, 8 (2012) p. 1981.

[60] A. Svete, M. Stefe, A. Macek, et al., Development of a measurement system for dynamic calibration of pressure sensors, in Proc. of the XXI IMEKO World Congress, 30 Aug. - 4 Sept. 2015, Prague, Czech Republic, pp. 1666-1671.

[61] A. Svete, M. Stefe, A. Macek, et al., Dynamic pressure generator for dynamic calibrations at different average pressures based on a double-acting pneumatic actuator, Sensors and Actuators A: Physical 247 (2016) p. 136.

[62] S. Razzak, J.-P. Damion, N. B. Hsouna, Dynamic pressure calibration, in Proc. of the XXI IMEKO World Congress, 30 Aug. -4 Sept. 2015, Prague, Czech Republic, p. 1660.

[63] Z. Zhang, W. Wang, Research on frequency response of pressure transducer, in Proc. of the XXI IMEKO World Congress, 30 Aug. - 4 Sept. 2015, Prague, Czech Republic, p. 1683.

[64] F. R. F. Theodoro, M. L. C. C. Reis, C. d'Andrade Souto, et al., Measurement uncertainty of a pressure sensor submitted to a step input, Measurement 88 (2016) p. 238.

[65] M. Kunimatsu, T. Mizuide, K. Yamane, Measurement of dynamic pressure using piezoelectric sensors at extremely low temperatures, JSAE Review 22, 4 (2001) p. 553.

[66] U. Rhyner, R. Mai, H. Leibold, et al., Dynamic pressure measurements of a hot gas filter as a diagnostic tool to assess the time dependent performance, Biomass and Bioenergy 53 (2013) p. 72.

[67] A. M. Hurst, J. VanDeWeert, A study of bulk modulus, entrained air, and dynamic pressure measurements in liquids, Journal of Engineering for Gas Turbines and Power 138, 10 (2016) p. 34.

[68] W. Shu, Dynamic weighing under nonzero initial condition, IEEE Transactions on Instrumentation and Measurement 42, 4 (1993) p. 806.

[69] E. Kaiser, Dynamic measuring error correction of encapsulated auxiliary wall heat flux sensor made of film resistance thermometer, Measurement 8, 1 (1990) p. 12

[70] S. Baglio, A. Bulsara, B. Audo, et al., Exploiting nonlinear dynamics in Novell measurement strategies and devices: from theory to experiments and applications, IEEE Transactions on Instrumentation and Measurement 60, 3 (2011) p. 667.

[71] A. Pardo, S. Marco, Nonlinear inverse dynamic models of gas sensing systems based on chemical sensor arrays for quantitative measurements, IEEE Transactions on Instrumentation and Measurement 47, 3 (1998) p. 644.

[72] J. R. Mejia, J. V. Arrovo, J. V. Pineda, et al., Comparison of compensation algorithms for smart sensors with approach to real-time or dynamic applications, IEEE Sensors Journal 15, 12 (2015) p. 7071.

[73] S. Saha, A new method using inverse theory for estimation of dynamic response in frequency domain for operation piping, Procedia Engineering 86 (2014) p. 843.

[74] A. Garcia, T. Hausotte, A. Amthor, Bayes filter for dynamic coordinate measurements: accuracy improvement, data fusion, and measurement uncertainty evaluation, Measurement 46, 9 (2013) p. 3734. 
[75] A. Javadi, J. Kragel, A. V. Makievski, et al., Fast interfacial tension measurements and dilational rheology of interfacial layers using the capillary pressure technique, Collaids and Surfaces A: Physiochemical and Engineering Aspects 407, 5 (2012) p. 159

[76] W. Zhu, Y. Deng, Y. Wang, Dynamic thermal response characteristics and feasibility analysis of thermoelectric module in impedance measurement, Applied Thermal Engineering 111, 25 (2017) p. 1417.

[77] K. Tomczyk, E. Layer, Accelerometer errors in the measurement if dynamic signals, Measurement 60 (2015) p. 2306.

[78] S. Yao, R. Gao, Nonlinear dynamic compensation research of temperature measurement system in coal mine movable refuge chamber, Procedia Engineering 2 (2011) p. 2306.
[79] R. Kozkmaz, B. A. Gozen, B. Bediz et al., High-frequency compensation of dynamic distortions in micromachine force measurements, Procedia Manufacturing 1 (2010) p. 534.

[80] K. E. Anders Ohlsson., R. Ostin, S. Gzundberg, et al., Dynamic model for measurement of convective heat transfer coefficient at external building surfaces, Journal of Building Engineering 7 (2016) p. 239.

[81] S. Eichstadt, C. Elster, I. M. Smith, T. J. Esward, Evaluation of dynamic measurement uncertainty: an open-source software package to bridge theory and practice, Journal of Sensors and Sensor Systems 6 (2017) p. 97.

[82] L. Klaus, T. Bruns, H. Volkers, Calibration of bridge-, chargeand voltage amplifiers for dynamic measurement applications, Metrologia 52, 1 (2015), 72- 81. WOS:000348119700011 